\title{
A New Method for the Stereoselective Synthesis of $\alpha$ - and $\beta$-Glycosylamines Using the Burgess Reagent
}

K. C. Nicolaou, * Scott A. Snyder, Annie Z. Nalbandian, Deborah A. Longbottom

Department of Chemistry and The Skaggs Institute for Chemical Biology

The Scripps Research Institute, 10550 North Torrey Pines Road, La Jolla, California 92037 and Department of Chemistry and Biochemistry, University of California, San Diego, 9500 Gilman Drive, La Jolla, California 92093

\section{Supporting Information}

\section{Experimental Data for Compounds}

General Procedures. All reactions were carried out under an argon atmosphere with dry solvents under anhydrous conditions, unless otherwise noted. Dry tetrahydrofuran (THF), benzene, and methylene chloride $\left(\mathrm{CH}_{2} \mathrm{Cl}_{2}\right)$ were obtained by passing commercially available predried, oxygen-free formulations through activated alumina columns. Yields refer to chromatographically and spectroscopically ( ${ }^{1} \mathrm{H}$ NMR) homogeneous materials, unless otherwise stated. Reagents were purchased at the highest commercial quality and used without further purification, unless otherwise stated. Reactions were monitored by thin-layer chromatography (TLC) carried out on $0.25 \mathrm{~mm}$ E. Merck silica gel plates (60F-254) using UV light as visualizing agent and an ethanolic solution of phosphomolybdic acid and cerium sulfate, and heat as developing agents. E. Merck silica gel (60, particle size $0.040-0.063 \mathrm{~mm})$ was used for flash column chromatography. NMR spectra were recorded on Bruker DRX-600, DRX-500, AMX500 or AMX-400 instruments and calibrated using residual undeuterated solvent as an internal reference. The following abbreviations were used to explain the multiplicities: $s=$ singlet, $d=$ doublet, $\mathrm{t}=$ triplet, $\mathrm{q}=$ quartet, $\mathrm{m}=$ multiplet, quin $=$ quintuplet, sext $=$ sextet, $\mathrm{sep}=$ septet, $\mathrm{br}=$ broad, $\mathrm{app}=$ apparent, $\mathrm{AB}=\mathrm{AB}$ quartet. IR spectra were recorded on a Perkin-Elmer 1600 series FT-IR spectrometer. Electrospray ionization (ESI) mass spectrometry (MS) experiments were 
performed on an API 100 Perkin Elmer SCIEX single quadrupole mass spectrometer at 4000V emitter voltage. High-resolution mass spectra (HRMS) were recorded on a VG ZAB-ZSE mass spectrometer using MALDI (matrix-assisted laser-desorption ionization).

General procedure for the synthesis of Burgess-type reagents. To a solution of chlorosulfonylisocyanate $\left(8.71 \mathrm{~mL}, 100 \mathrm{mmol}, 1.0\right.$ equiv) in $\mathrm{CH}_{2} \mathrm{Cl}_{2}(25 \mathrm{~mL})$ at $0{ }^{\circ} \mathrm{C}$ was added a solution of the appropriate alcohol (105 mmol, 1.05 equiv) in $\mathrm{CH}_{2} \mathrm{Cl}_{2}(25 \mathrm{~mL})$ over the course of 30 minutes. Once the addition was complete, the reaction contents were concentrated directly to give the desired sulfamoyl chloride intermediate as a white solid. Pressing forward without any additional purification steps, a solution of the newly-formed sulfamoyl chloride $(20.0 \mathrm{mmol}$, 1.0 equiv) in benzene $(40 \mathrm{~mL})$ was added dropwise to a solution of $\mathrm{Et}_{3} \mathrm{~N}(6.27 \mathrm{~mL}, 45 \mathrm{mmol}$, 2.25 equiv) in benzene $(25 \mathrm{~mL})$ at $25{ }^{\circ} \mathrm{C}$. After the addition was complete ( $\sim 1$ hour $)$, the triethylammonium hydrochloride precipitate was removed by filtration, and the filtrate was concentrated to give $\mathbf{1}, \mathbf{2}$, or $\mathbf{3}$ as clear oils which solidified upon standing.

Alloc-protected Burgess-type reagent 2: $\mathrm{R}_{f}=0.04$ (silica gel, EtOAc); IR (film) $v_{\max }$ 2995, 2948, 1693, 1457, 1332, 1161, 1098, 970, 859, 788, 714, 597, $548 \mathrm{~cm}^{-1} ;{ }^{1} \mathrm{H}$ NMR (500 $\left.\mathrm{MHz}, \mathrm{CDCl}_{3}\right) \delta 5.91(\mathrm{~m}, 1 \mathrm{H}), 5.30(\mathrm{~d}, J=16.2 \mathrm{~Hz}, 1 \mathrm{H}), 5.18(\mathrm{~d}, J=10.3 \mathrm{~Hz}, 1 \mathrm{H}), 4.54(\mathrm{~s}, 2$ $\mathrm{H}), 3.45(\mathrm{q}, J=7.4 \mathrm{~Hz}, 6 \mathrm{H}), 1.39(\mathrm{t}, J=7.0 \mathrm{~Hz}, 9 \mathrm{H}) ;{ }^{13} \mathrm{C} \mathrm{NMR}\left(125 \mathrm{MHz}, \mathrm{CDCl}_{3}\right) \delta 157.2$, 132.5, 117.9, 66.6, 50.5, 9.3; MS (ESI) calcd for $\mathrm{C}_{10} \mathrm{H}_{21} \mathrm{~N}_{2} \mathrm{O}_{4} \mathrm{~S}^{+}\left[\mathrm{M}+\mathrm{H}^{+}\right] 265$, found 265 .

Troc-protected Burgess-type reagent 3: $\mathrm{R}_{f}=0.03$ (silica gel, EtOAc); IR (film) $v_{\max }$ 2950, 1702, 1457, 1368, 1339, 1254, 1111, 1059, 857, 816, 716, 605, $548 \mathrm{~cm}^{-1} ;{ }^{1} \mathrm{H}$ NMR (400 $\left.\mathrm{MHz}, \mathrm{CDCl}_{3}\right) \delta 4.75(\mathrm{~s}, 2 \mathrm{H}), 3.48(\mathrm{q}, J=7.4 \mathrm{~Hz}, 6 \mathrm{H}), 1.43(\mathrm{t}, J=7.3 \mathrm{~Hz}, 9 \mathrm{H}) ;{ }^{13} \mathrm{C}$ NMR $(100$ $\left.\mathrm{MHz}, \mathrm{CDCl}_{3}\right) \delta 156.0,95.4,75.3,50.7,9.3$; MS (ESI) calcd for $\mathrm{C}_{9} \mathrm{H}_{18} \mathrm{Cl}_{3} \mathrm{~N}_{2} \mathrm{O}_{4} \mathrm{~S}^{+}\left[\mathrm{M}+\mathrm{H}^{+}\right] 355$, found 355 .

3,4,6-Tri-O-benzyl-D-glucopyranose 12. 4-Methylmorpholine $N$-oxide $(0.351 \mathrm{~g}, 3.0$ mmol, 3.0 equiv) and osmium tetroxide $(0.641 \mathrm{~mL}, 2.5 \mathrm{wt} \%$ in $t$ - $\mathrm{BuOH}, 0.05 \mathrm{mmol}, 0.05$ equiv) were added sequentially at $25^{\circ} \mathrm{C}$ to a solution of 3,4,6-tri- $O$-benzyl-D-glucal $(0.417 \mathrm{~g}, 1.0 \mathrm{mmol}$, 
1.0 equiv) in $\mathrm{THF} / t$ - $\mathrm{BuOH} / \mathrm{H}_{2} \mathrm{O}(7: 3: 1,5 \mathrm{ml})$, and the resulting orange solution was stirred for 12 hours at $25{ }^{\circ} \mathrm{C}$. Upon completion, the reaction contents were then diluted with water $(10 \mathrm{~mL})$, treated with $\mathrm{Na}_{2} \mathrm{SO}_{3}(0.630 \mathrm{~g}, 5.0 \mathrm{mmol}, 5.0$ equiv), and allowed to stir for an additional 2 hours at $25^{\circ} \mathrm{C}$. Once this operation was complete, the reaction mixture was poured into water $(10 \mathrm{~mL})$ and extracted with EtOAc $(3 \times 25 \mathrm{~mL})$. The combined organic layers were then washed with water $(2 \times 15 \mathrm{~mL})$, dried $\left(\mathrm{MgSO}_{4}\right)$, and concentrated. The resultant light yellow solid was purified by flash column chromatography (silica gel, EtOAc/hexanes, 1:1) to give diol $12(0.414$ g, 92\%) as an amorphous white solid. 12: $\mathrm{R}_{f}=0.13$ (silica gel, EtOAc/hexanes, 1:1); IR (film) $v_{\max } 3410,2938,1452,1390,1190,1080,886,590 \mathrm{~cm}^{-1} ;{ }^{1} \mathrm{H}$ NMR $\left(500 \mathrm{MHz}, \mathrm{CDCl}_{3}, 1: 1\right.$ mixture of anomers) $\delta 7.38-7.10(\mathrm{~m}, 30 \mathrm{H}), 5.18(\mathrm{~d}, J=4.8 \mathrm{~Hz}, 1 \mathrm{H}), 4.81(\mathrm{~s}, 2 \mathrm{H}), 4.75(\mathrm{~d}, J=$ $13.9 \mathrm{~Hz}, 2 \mathrm{H}), 4.54-4.49$ (m, $3 \mathrm{H}), 4.47$ (app t, $J=5.5 \mathrm{~Hz}, 3 \mathrm{H}), 4.43$ (d, $J=3.3 \mathrm{~Hz}, 1 \mathrm{H}), 4.01$ (t, $J=6.4 \mathrm{~Hz}, 1 \mathrm{H}), 3.98(\mathrm{dd}, J=6.2 \mathrm{~Hz}, 1 \mathrm{H}), 3.74($ app t, $J=11.4 \mathrm{~Hz}, 1 \mathrm{H}), 3.63-3.58(\mathrm{~m}, 3$ $\mathrm{H}), 3.51-3.46(\mathrm{~m}, 2 \mathrm{H}) ;{ }^{13} \mathrm{C} \mathrm{NMR}\left(125 \mathrm{MHz}, \mathrm{CDCl}_{3}, 1: 1\right.$ mixture of anomers) $\delta 138.5,138.4$, $138.0,137.8,137.6,128.5,128.4,128.0,127.9,127.8,127.7,96.7,92.3,84.3,82.5,77.6,77.5$, 75.5, 75.3, 74.8, 73.4, 72.7, 70.4, 68.7; HRMS (MALDI-FTMS) calcd for $\mathrm{C}_{27} \mathrm{H}_{30} \mathrm{O}_{6} \mathrm{Na}^{+}[\mathrm{M}+$ $\left.\mathrm{Na}^{+}\right]$473.1934, found 473.1925 .

3,4-Di-O-benzyl-L-rhamnopyranose 16. 4-O-Benzyl-L-rhamnal $(0.220 \mathrm{~g}, 1.0 \mathrm{mmol}$, 1.0 equiv) was dissolved in DMF $(5 \mathrm{~mL})$ and treated with $\mathrm{NaH}(0.400 \mathrm{~g}, 60 \%$ dispersion in mineral oil, $2.0 \mathrm{mmol}, 2.0$ equiv) at $25{ }^{\circ} \mathrm{C}$. After stirring the resultant solution for 5 minutes at $25^{\circ} \mathrm{C}$, the reaction contents were cooled to $0{ }^{\circ} \mathrm{C}$ and benzyl bromide $(0.238 \mathrm{~mL}, 2.0 \mathrm{mmol}, 2.0$ equiv) added dropwise over the course of 10 minutes. The reaction mixture was then warmed to $25{ }^{\circ} \mathrm{C}$ over the course of 30 minutes. Upon completion, the reaction mixture was poured into $1 \mathrm{~N}$ aqueous $\mathrm{HCl}(10 \mathrm{~mL})$ and extracted with $\mathrm{Et}_{2} \mathrm{O}(3 \times 25 \mathrm{~mL})$. The combined organic layers were then washed with water $(2 \times 15 \mathrm{~mL})$, dried $\left(\mathrm{MgSO}_{4}\right)$, and concentrated. The resultant light yellow solid was purified by flash column chromatography (silica gel, $\mathrm{Et}_{2} \mathrm{O}$ ) to give the desired di-O-benzylated-L-rhamnal intermediate $(0.292 \mathrm{~g}, 94 \%)$ as an amorphous white solid. This compound was then dihydroxylated in a manner similar to the preparation of $\mathbf{1 2}$ to give diol $\mathbf{1 6}$ 
(0.314 g, 91\% yield) as a white amorphous solid. 16: $\mathrm{R}_{f}=0.40$ (silica gel, EtOAc/hexanes, 1:1); IR (film) $v_{\max } 3388,2870,1645,1453,1363,1091,994,734,694 \mathrm{~cm}^{-1} ;{ }^{1} \mathrm{H}$ NMR (500 MHz, $\mathrm{CDCl}_{3}, 1: 1$ mixture of anomers) $\delta 7.40-7.32(\mathrm{~m}, 20 \mathrm{H}), 5.34(\mathrm{~d}, J=3.0 \mathrm{~Hz}, 1 \mathrm{H}), 4.98(\mathrm{~d}, J=2.6$ Hz, $1 \mathrm{H}), 4.95(\mathrm{~d}, J=2.6 \mathrm{~Hz}, 1 \mathrm{H}), 4.77$ (br d, $J=8.4 \mathrm{~Hz}, 1 \mathrm{H}), 4.71-4.62$ (m, $5 \mathrm{H}), 4.04$ (m, 2 H), 3.64 (ddd, $J=13.1,8.5,4.8 \mathrm{~Hz}, 1 \mathrm{H}), 3.40$ (dd, $J=9.6,6.3 \mathrm{~Hz}, 1 \mathrm{H}), 3.21$ (br s, $1 \mathrm{H}), 3.15$ (dt, $J=8.7,3.7 \mathrm{~Hz}, 1 \mathrm{H}), 2.60$ (br s), 2.43 (ddd, $J=12.5,5.2,2.2 \mathrm{~Hz}, 1 \mathrm{H}), 2.32$ (ddd, $J=13.2$, 5.2, $1.5 \mathrm{~Hz}, 1 \mathrm{H}), 1.74(\mathrm{~m}, 1 \mathrm{H}), 1.43(\mathrm{~m}, 1 \mathrm{H}), 1.35(\mathrm{~d}, J=6.3 \mathrm{~Hz}, 3 \mathrm{H}), 1.28(\mathrm{~d}, J=6.3 \mathrm{~Hz}, 3$ $\mathrm{H}) ;{ }^{13} \mathrm{C} \mathrm{NMR}\left(125 \mathrm{MHz}, \mathrm{CDCl}_{3}, 1: 1\right.$ mixture of anomers) $\delta 138.6,138.5,138.3,138.2,128.4(3$ C), 128.3, 128.0 (2 C), 127.7 (2 C), 127.6 (2 C), 93.8, 92.0, 84.3, 83.4, 78.9, 76.8, 75.2 (2 C), 71.8 (2 C), 71.5, 71.4, 67.4, 38.3, 37.7, 18.2 (2 C); MS (ESI) calcd for $\mathrm{C}_{20} \mathrm{H}_{22} \mathrm{O}_{4}{ }^{+}\left[\mathrm{M}-\mathrm{H}_{2} \mathrm{O}^{+}\right]$ 327 , found 327.

3,4,6-Tri- $O$-benzyl-D-galactopyranose 18 . 3,4,6-Tri- $O$-benzyl- $\beta$-D-galactal $(0.417 \mathrm{~g}$, $1.0 \mathrm{mmol}, 1.0$ equiv) was dihydroxylated in a manner similar to the preparation of $\mathbf{1 2}$ to give diol $18\left(0.379 \mathrm{~g}, 91 \%\right.$ yield) as a white amorphous solid. 18: $\mathrm{R}_{f}=0.13$ (silica gel, EtOAc/hexanes, 1:1); IR (film) $v_{\max } 3401,2919,2861,1455,1361,1085,738,691 \mathrm{~cm}^{-1} ;{ }^{1} \mathrm{H}$ NMR (400 MHz, $\left.\mathrm{CDCl}_{3}\right) \delta 7.41-7.26(\mathrm{~m}, 15 \mathrm{H}), 5.31(\mathrm{~d}, J=3.5 \mathrm{~Hz}, 1 \mathrm{H}), 4.87(\mathrm{dd}, J=11.8,3.5 \mathrm{~Hz}, 1 \mathrm{H}), 4.70$ (m, $2 \mathrm{H}), 4.61-4.39(\mathrm{~m}, 4 \mathrm{H}), 4.14(\mathrm{dd}, J=10.0,3.8 \mathrm{~Hz}, 1 \mathrm{H}), 3.88$ (m, $1 \mathrm{H}), 3.73$ (dd, $J=10.0$, $2.6 \mathrm{~Hz}, 1 \mathrm{H}), 3.61-3.50(\mathrm{~m}, 3 \mathrm{H}), 3.41(\mathrm{~m}, 1 \mathrm{H}) ;{ }^{13} \mathrm{C} \mathrm{NMR}\left(100 \mathrm{MHz}, \mathrm{CDCl}_{3}\right) \delta 138.3,138.0$, 137.6, 128.5, 128.4, 128.2, 128.0 (2 C), 127.8, 127.7, 92.6, 79.0, 74.5, 73.8, 73.4, 72.3, 69.6, 69.2, 68.9; HRMS (MALDI-FTMS) calcd for $\mathrm{C}_{27} \mathrm{H}_{30} \mathrm{O}_{6} \mathrm{Na}^{+}\left[\mathrm{M}+\mathrm{Na}^{+}\right]$473.1934, found 473.1921.

3,5-Di-O-TBS-D-ribofuranose 22. The requisite D-ribose glycal intermediate was prepared from thymidine following the procedure of Larsen and co-workers. ${ }^{1}$ The resultant product was then dihydroxylated in a manner similar to the preparation of $\mathbf{1 2}$ to give diol 22 (0.378 g, 88\% yield) as a white amorphous solid. 22: $\mathrm{R}_{f}=0.35$ (silica gel, EtOAc/hexanes, 1:2); IR (film) $v_{\max } 3425,2930,2858,1471,1391,1255,1095,937,779 \mathrm{~cm}^{-1} ;{ }^{1} \mathrm{H}$ NMR (400 MHz, $\mathrm{CDCl}_{3}, 1: 1$ mixture of anomers) $\delta 5.24(\mathrm{~d}, J=6.8 \mathrm{~Hz}, 1 \mathrm{H}), 5.13(\mathrm{~d}, J=10.6 \mathrm{~Hz}, 1 \mathrm{H}), 4.24$ (br 
$\mathrm{d}, J=8.5 \mathrm{~Hz}, 1 \mathrm{H}), 4.18(\operatorname{app~t}, J=2.6 \mathrm{~Hz}, 1 \mathrm{H}), 4.09-4.06(\mathrm{~m}, 2 \mathrm{H}), 3.91$ (br d, $J=11.1 \mathrm{~Hz}, 1$ H), 3.86-3.81 (m, $2 \mathrm{H}), 3.77(\mathrm{dd}, J=8.6,2.6 \mathrm{~Hz}, 1 \mathrm{H}), 3.71(\mathrm{~d}, J=1.8 \mathrm{~Hz}, 1 \mathrm{H}), 3.63(\mathrm{dd}, J=$ 10.8, 2.0 Hz, $1 \mathrm{H}), 0.89$ (s, $9 \mathrm{H}), 0.87$ (s, $9 \mathrm{H}), 0.84$ (s, $9 \mathrm{H}), 0.82$ (s, $9 \mathrm{H}), 0.10$ (s, $6 \mathrm{H}), 0.07-$ $0.04(\mathrm{~m}, 18 \mathrm{H}) ;{ }^{13} \mathrm{C} \mathrm{NMR}\left(100 \mathrm{MHz}, \mathrm{CDCl}_{3}, 1: 1\right.$ mixture of anomers $) \delta 104.1,97.6,87.1,84.8$, $78.8,78.5,77.3,69.9,65.7,63.5,63.1,25.7,25.5,17.7,15.1,-4.7,-5.0,-5.1,-5.6,-5.7,-5.8$; HRMS (MALDI-FTMS) calcd for $\mathrm{C}_{17} \mathrm{H}_{38} \mathrm{O}_{5} \mathrm{Si}_{2} \mathrm{Na}^{+}\left[\mathrm{M}+\mathrm{Na}^{+}\right]$401.2150, found 401.2147.

General procedure for the synthesis of sulfamidates on carbohydrates. The appropriate carbohydrate diol (0.5 mmol, 1.0 equiv) was dissolved in $\mathrm{THF} / \mathrm{CH}_{2} \mathrm{Cl}_{2}(4: 1,5 \mathrm{~mL})$ and the desired Burgess-type reagent (1, 2 or 3, 1.25 mmol, 2.5 equiv) was added at $25^{\circ} \mathrm{C}$ in a single portion. The resultant solution was immediately warmed to reflux (using a preheated oil bath) and stirred for 6 hours. Upon completion, the reaction contents were cooled to $25{ }^{\circ} \mathrm{C}$, poured into saturated aqueous $\mathrm{NH}_{4} \mathrm{Cl}(25 \mathrm{~mL})$, and extracted with $\mathrm{CH}_{2} \mathrm{Cl}_{2}(3 \times 25 \mathrm{~mL})$. The combined organic layers were then washed with water $(50 \mathrm{~mL})$, dried $\left(\mathrm{MgSO}_{4}\right)$, and concentrated. The resultant yellow residue was purified by flash column chromatography (silica gel) in an appropriate solvent system to give the desired product in high purity.

13: $\mathrm{R}_{f}=0.68$ (silica gel, EtOAc/hexanes, 1:1); IR (film) $v_{\max } 3037,2866,1753,1444$, 1385, 1312, 1196, 1094, 842, 741, 699, $665 \mathrm{~cm}^{-1} ;{ }^{1} \mathrm{H}$ NMR (600 MHz, $\left.\mathrm{CDCl}_{3}\right) \delta$ 7.39-7.29 (m, $13 \mathrm{H}), 7.21(\mathrm{~m}, 2 \mathrm{H}), 6.02(\mathrm{~d}, J=4.8 \mathrm{~Hz}, 1 \mathrm{H}), 4.90(\mathrm{t}, J=4.0 \mathrm{~Hz}, 1 \mathrm{H}), 4.60(\mathrm{~s}, 2 \mathrm{H}), 4.47$ (AB, $\left.J=11.8 \mathrm{~Hz}, \mathrm{v}_{\mathrm{ab}}=88.6 \mathrm{~Hz}, 2 \mathrm{H}\right), 4.44\left(\mathrm{AB}, J=11.9 \mathrm{~Hz}, \mathrm{v}_{\mathrm{ab}}=88.6 \mathrm{~Hz}, 2 \mathrm{H}\right), 4.09(\mathrm{t}, J=3.5 \mathrm{~Hz}$, $1 \mathrm{H}), 3.96(\mathrm{dd}, J=11.4,2.6 \mathrm{~Hz}, 1 \mathrm{H}), 3.94(\mathrm{~s}, 3 \mathrm{H}), 3.92(\mathrm{dd}, J=9.2,3.5 \mathrm{~Hz}, 1 \mathrm{H}), 3.68(\mathrm{dd}, J=$ 11.0, $2.2 \mathrm{~Hz}, 1 \mathrm{H}), 3.61(\mathrm{dd}, J=10.9,3.5 \mathrm{~Hz}, 1 \mathrm{H}) ;{ }^{13} \mathrm{C} \mathrm{NMR}\left(150 \mathrm{MHz}, \mathrm{CDCl}_{3}\right) \delta 149.8,137.8$, $137.3,136.6,128.7,128.5,128.4,128.3,128.1,128.0$ (2 C), 127.8, 127.7, 81.6, 75.5, 73.3, 73.1, 72.9, 72.4, 68.9, 54.9; HRMS (MALDI-FTMS) calcd for $\mathrm{C}_{29} \mathrm{H}_{31} \mathrm{NO}_{9} \mathrm{SNa}^{+}\left[\mathrm{M}+\mathrm{Na}^{+}\right]$592.1612, found 592.1608 .

14: $\mathrm{R}_{f}=0.67$ (silica gel, EtOAc/hexanes, 1:1); IR (film) $v_{\max } 3363,2923,1763,1453$, 1390, 1294, 1199, 1092, 847, 738, $699 \mathrm{~cm}^{-1} ;{ }^{1} \mathrm{H}$ NMR (500 MHz, $\left.\mathrm{CDCl}_{3}\right) \delta$ 7.30-7.20 (m, 15 H), $6.09(\mathrm{~d}, J=5.2 \mathrm{~Hz}, 1 \mathrm{H}), 4.95(\mathrm{~d}, J=4.4 \mathrm{~Hz}, 1 \mathrm{H}), 4.90\left(\mathrm{AB}, J=12.1 \mathrm{~Hz}, \mathrm{v}_{\mathrm{ab}}=48.4 \mathrm{~Hz}, 2\right.$ 
H), $4.62(\mathrm{~s}, 2 \mathrm{H}), 4.45\left(\mathrm{AB}, J=11.7 \mathrm{~Hz}, \mathrm{v}_{\mathrm{ab}}=101.2 \mathrm{~Hz}, 2 \mathrm{H}\right), 4.43\left(\mathrm{AB}, J=11.8 \mathrm{~Hz}, \mathrm{v}_{\mathrm{ab}}=44.8\right.$ Hz, $2 \mathrm{H}), 4.12$ (t, $J=3.7 \mathrm{~Hz}, 1 \mathrm{H}), 3.99$ (dt, $J=9.2,2.6 \mathrm{~Hz}, 1 \mathrm{H}), 3.93$ (dd, $J=9.2,3.3 \mathrm{~Hz}, 1 \mathrm{H})$, $3.68(\mathrm{dd}, J=11.0,2.2 \mathrm{~Hz}, 1 \mathrm{H}), 3.61(\mathrm{dd}, J=11.0,3.3 \mathrm{~Hz}, 1 \mathrm{H}) ;{ }^{13} \mathrm{C} \mathrm{NMR}\left(125 \mathrm{MHz}, \mathrm{CDCl}_{3}\right) \delta$ $148.1,137.8,137.3,136.6,128.7,128.5,128.4,128.1,128.0,127.8,127.7,93.6,81.8,76.0,75.4$, 73.4, 73.3, 72.9, 72.8, 72.5, 68.8; HRMS (MALDI-FTMS) calcd for $\mathrm{C}_{30} \mathrm{H}_{30} \mathrm{Cl}_{3} \mathrm{NO}_{9} \mathrm{SNa}^{+}[\mathrm{M}+$ $\left.\mathrm{Na}^{+}\right]$708.0599, found 708.0611.

15: $\mathrm{R}_{f}=0.68$ (silica gel, EtOAc/hexanes, 1:1); IR (film) $v_{\max } 3398,2865,1746,1453$, 1376, 1300, 1284, 1197, 1088, $1028 \mathrm{~cm}^{-1} ;{ }^{1} \mathrm{H}$ NMR $\left(500 \mathrm{MHz}, \mathrm{CDCl}_{3}\right) \delta 7.36-7.29$ (m, $\left.13 \mathrm{H}\right)$, 7.20-7.18 (m $2 \mathrm{H}), 6.02$ (d, $J=4.8 \mathrm{~Hz}, 1 \mathrm{H}), 5.93$ (m, $1 \mathrm{H}), 5.42$ (dd, $J=17.0,1.1 \mathrm{~Hz}, 1 \mathrm{H}), 5.30$ $(\mathrm{m}, 1 \mathrm{H}), 4.88(\mathrm{dt}, J=8.1,0.7 \mathrm{~Hz}, 1 \mathrm{H}), 4.79\left(\mathrm{ABX}, J=16.1,5.5 \mathrm{~Hz}, \mathrm{v}_{\mathrm{ab}}=21.6 \mathrm{~Hz}, 2 \mathrm{H}\right), 4.60$ $(\mathrm{s}, 2 \mathrm{H}), 4.53\left(\mathrm{AB}, J=12.1 \mathrm{~Hz}, \mathrm{v}_{\mathrm{ab}}=21.3 \mathrm{~Hz}, 2 \mathrm{H}\right), 4.38\left(\mathrm{AB}, J=12.1 \mathrm{~Hz}, \mathrm{v}_{\mathrm{ab}}=22.4 \mathrm{~Hz}, 2 \mathrm{H}\right)$, $4.08(\mathrm{t}, J=3.7 \mathrm{~Hz}, 1 \mathrm{H}), 3.95(\mathrm{td}, J=7.4,2.8 \mathrm{~Hz}, 1 \mathrm{H}), 3.89$ (dd, $J=7.4,3.7 \mathrm{~Hz}, 1 \mathrm{H}), 3.67$ (dd, $J=9.3,2.2 \mathrm{~Hz}, 1 \mathrm{H}), 3.59(\mathrm{dd}, J=9.1,3.7 \mathrm{~Hz}, 1 \mathrm{H}) ;{ }^{13} \mathrm{C} \mathrm{NMR}\left(125 \mathrm{MHz}, \mathrm{CDCl}_{3}\right) \delta 149.1$, 137.9, 137.4, 136.7, 31.4, 130.4, 128.7, 128.5, 128.4 (2 C), 128.1 (2 C), 127.9, 127.7, 119.6, 119.2, 81.6, 77.4, 75.6, 73.5, 73.4, 72.9, 72.8, 72.4, 69.0, 68.5, 66.8; HRMS (MALDI-FTMS) calcd for $\mathrm{C}_{31} \mathrm{H}_{33} \mathrm{NO}_{9} \mathrm{SNa}^{+}\left[\mathrm{M}+\mathrm{Na}^{+}\right]$618.1768, found 618.1773.

17: $\mathrm{R}_{f}=0.60$ (silica gel, EtOAc/hexanes, 1:1); IR (film) $v_{\max } 3248,1752,1636,1494$, $1444,1381,1314,1196,1146,1077,1001,740 \mathrm{~cm}^{-1} ;{ }^{1} \mathrm{H}$ NMR $\left(400 \mathrm{MHz}, \mathrm{CDCl}_{3}\right) \delta$ 7.41-7.29 (m, 8 H), 7.27-7.25 (m, $2 \mathrm{H}), 5.88$ (d, $J=5.3 \mathrm{~Hz}, 1 \mathrm{H}), 4.90$ (ddd, $J=6.2,3.5,1.2 \mathrm{~Hz}, 1 \mathrm{H}), 4.57$ $\left(\mathrm{AB}, J=10.8 \mathrm{~Hz}, v_{\mathrm{ab}}=11.8 \mathrm{~Hz}, 2 \mathrm{H}\right), 4.44\left(\mathrm{AB}, J=12.0 \mathrm{~Hz}, v_{\mathrm{ab}}=34.9 \mathrm{~Hz}, 2 \mathrm{H}\right), 4.02(\mathrm{dd}, J=$ 3.2, 2.0, Hz, $1 \mathrm{H}), 3.98$ (m, $1 \mathrm{H}), 3.95$ (s, $3 \mathrm{H}), 3.36$ (ddd, $J=8.8,1.8,1.2 \mathrm{~Hz}, 1 \mathrm{H}), 1.28$ (d, $J=$ $6.2 \mathrm{~Hz}, 3 \mathrm{H}) ;{ }^{13} \mathrm{C}$ NMR $\left(100 \mathrm{MHz}, \mathrm{CDCl}_{3}\right) \delta 150.0,137.3,136.5,128.8,128.5(2 \mathrm{C}), 128.1$, 128.0 (3 C), 81.5, 80.5, 75.2, 74.4, 72.6, 72.4, 67.9, 54.9, 19.3; HRMS (MALDI-FTMS) calcd for $\mathrm{C}_{22} \mathrm{H}_{25} \mathrm{NO}_{8} \mathrm{SNa}^{+}\left[\mathrm{M}+\mathrm{Na}^{+}\right] 486.1193$, found 486.1202 .

19: $\mathrm{R}_{f}=0.66$ (silica gel, EtOAc/hexanes, 1:1); IR (film) $v_{\max }$ 2961, 1755, 1443, 1384, 1320, 1290, 1190, 1061, 991, 855, 732, $697 \mathrm{~cm}^{-1} ;{ }^{1} \mathrm{H}$ NMR (600 MHz, $\left.\mathrm{CDCl}_{3}\right) \delta$ 7.39-7.25 (m, $15 \mathrm{H}), 5.89(\mathrm{~d}, J=4.0 \mathrm{~Hz}, 1 \mathrm{H}), 5.02(\mathrm{dd}, J=5.2,3.5 \mathrm{~Hz}, 1 \mathrm{H}), 4.75\left(\mathrm{AB}, J=11.0 \mathrm{~Hz}, \mathrm{v}_{\mathrm{ab}}=\right.$ 
$160.4 \mathrm{~Hz}, 2 \mathrm{H}), 4.73\left(\mathrm{AB}, J=11.9 \mathrm{~Hz}, \mathrm{v}_{\mathrm{ab}}=68.8 \mathrm{~Hz}, 2 \mathrm{H}\right), 4.53\left(\mathrm{AB}, J=11.9 \mathrm{~Hz}, \mathrm{v}_{\mathrm{ab}}=31.1\right.$ Hz, $2 \mathrm{H}), 4.20(\mathrm{~m}, 1 \mathrm{H}), 4.15(\mathrm{dd}, J=4.0,1.7 \mathrm{~Hz}, 1 \mathrm{H}), 4.03(\mathrm{dd}, J=5.3,1.8 \mathrm{~Hz}, 1 \mathrm{H}), 3.92(\mathrm{~s}, 3$ $\mathrm{H}), 3.71(\mathrm{dd}, J=9.7,7.4 \mathrm{~Hz}, 1 \mathrm{H}), 3.65(\mathrm{dd}, J=9.7,6.5 \mathrm{~Hz}, 1 \mathrm{H}) ;{ }^{13} \mathrm{C} \mathrm{NMR}\left(150 \mathrm{MHz}, \mathrm{CDCl}_{3}\right)$ $\delta 149.5,137.7,137.4,136.8,128.6,128.4,128.2,128.1,128.0,127.8$ (2 C), 127.6, 84.1, 80.3, 78.7, 75.4, 75.3, 73.7, 72.1, 66.9, 54.9; HRMS (MALDI-FTMS) calcd for $\mathrm{C}_{29} \mathrm{H}_{31} \mathrm{NO}_{9} \mathrm{SNa}^{+}[\mathrm{M}+$ $\left.\mathrm{Na}^{+}\right]$592.1612, found 592.1633.

21: $\mathrm{R}_{f}=0.33$ (silica gel, EtOAc/hexanes, 1:2); IR (film) $\mathrm{v}_{\max } 2978,1746,1437,1412$, 1294, 1249, 1187, 1073, 1023, 973, 861, 797, $609 \mathrm{~cm}^{-1} ;{ }^{1} \mathrm{H}$ NMR (400 MHz, $\left.\mathrm{CDCl}_{3}\right) \delta 5.98(\mathrm{~d}, J$ $=3.8 \mathrm{~Hz}, 1 \mathrm{H}), 5.13(\mathrm{~d}, J=2.4 \mathrm{~Hz}, 1 \mathrm{H}), 4.72(\mathrm{dd}, J=15.0,2.9 \mathrm{~Hz}, 1 \mathrm{H}), 4.71(\mathrm{~d}, J=3.5 \mathrm{~Hz}, 1$ H), 4.36 (q, $J=2.6 \mathrm{~Hz}, 1 \mathrm{H}), 4.09$ (dd, $J=15.0,2.6 \mathrm{~Hz}, 1 \mathrm{H}), 3.86$ (s, $3 \mathrm{H}), 1.49$ (s, $3 \mathrm{H}), 1.33$ (s, $3 \mathrm{H}) ;{ }^{13} \mathrm{C} \mathrm{NMR}\left(100 \mathrm{MHz}, \mathrm{CDCl}_{3}\right) \delta 151.9,113.0,104.9,87.3,82.5,70.6,54.9,48.2,26.5,26.1$; HRMS (MALDI-FTMS) calcd for $\mathrm{C}_{10} \mathrm{H}_{15} \mathrm{NO}_{8} \mathrm{SNa}^{+}\left[\mathrm{M}+\mathrm{Na}^{+}\right]$332.0411, found 332.0418.

23: $\mathrm{R}_{f}=0.68$ (silica gel, EtOAc/hexanes, 1:2); IR (film) $\mathrm{v}_{\max } 2954,2858,1759,1442$, 1392, 1313, 1257, 1196, 1098, 1011, 838, 806, $781 \mathrm{~cm}^{-1} ;{ }^{1} \mathrm{H}$ NMR $\left(500 \mathrm{MHz}, \mathrm{CDCl}_{3}\right) \delta 6.12(\mathrm{~d}$, $J=4.4 \mathrm{~Hz}, 1 \mathrm{H}), 4.93(\mathrm{~d}, J=4.1 \mathrm{~Hz}, 1 \mathrm{H}), 4.61(\mathrm{~s}, 1 \mathrm{H}), 4.12(\mathrm{ddd}, J=8.5,5.2,1.9 \mathrm{~Hz}, 1 \mathrm{H})$, $3.96(\mathrm{~s}, 3 \mathrm{H}), 3.77$ (dd, $J=10.7,5.5 \mathrm{~Hz}, 1 \mathrm{H}), 3.71(\mathrm{dd}, J=10.6,8.8 \mathrm{~Hz}, 1 \mathrm{H}), 0.90(\mathrm{~s}, 18 \mathrm{H})$, $0.14(\mathrm{~d}, J=5.5 \mathrm{~Hz}, 6 \mathrm{H}), 0.07(\mathrm{~d}, J=2.6 \mathrm{~Hz}, 6 \mathrm{H}) ;{ }^{13} \mathrm{C} \mathrm{NMR}\left(125 \mathrm{MHz}, \mathrm{CDCl}_{3}\right) \delta 149.8,88.8$, $87.7,86.2,74.3,61.7,55.0,25.8,25.5,18.3,17.9,-4.9,-5.0,-5.5$ (2 C); HRMS (MALDIFTMS) calcd for $\mathrm{C}_{19} \mathrm{H}_{40} \mathrm{NO}_{8} \mathrm{Si}_{2} \mathrm{~S}^{+}\left[\mathrm{M}+\mathrm{H}^{+}\right]$498.2008, found 498.2015.

Scheme S1. X-ray crystallographic proof of sulfamidate stereochemistry.
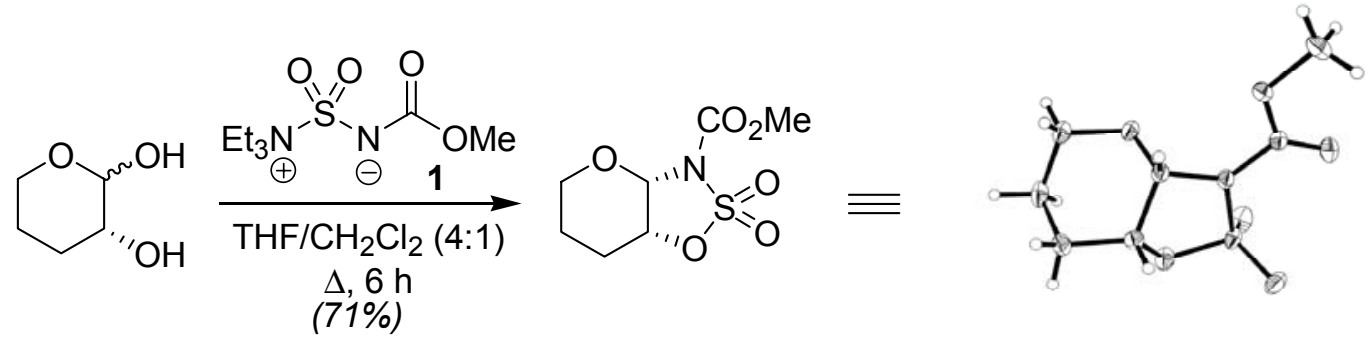
2-Azido-3,4,6-tri-O-benzyl-D-glucopyranose 24. $\mathrm{NaN}_{3}(0.033$ g, 0.50 mmol, 5.0 equiv) was added in a single portion to a solution of glucose-derived sulfamidate $13(0.057 \mathrm{~g}, 0.10$ mmol, 1.0 equiv) in $\mathrm{THF} / \mathrm{CH}_{2} \mathrm{Cl}_{2}(4: 1,1 \mathrm{ml})$ at $25{ }^{\circ} \mathrm{C}$. The resulting clear solution was then warmed to $60{ }^{\circ} \mathrm{C}$ and stirred for 5 hours. Upon completion, the reaction contents were diluted with $\mathrm{Et}_{2} \mathrm{O}(5 \mathrm{~mL})$, treated with $10 \%$ aqueous $\mathrm{H}_{2} \mathrm{SO}_{4}(1 \mathrm{~mL})$, and allowed to stir for an additional 30 minutes at $25^{\circ} \mathrm{C}$. Once this operation was complete, the reaction mixture was poured into water $(10 \mathrm{~mL})$ and extracted with $\mathrm{Et}_{2} \mathrm{O}(3 \times 25 \mathrm{~mL})$. The combined organic layers were then washed with water $(2 \times 15 \mathrm{~mL})$, dried $\left(\mathrm{MgSO}_{4}\right)$, and concentrated. The resultant light yellow solid was purified by flash column chromatography (silica gel, $\mathrm{Et}_{2} \mathrm{O} /$ hexanes, $1: 1$ ) to give the azide-opened tri-benzylated glucose derivative $24(0.049 \mathrm{~g}, 92 \%)$ as an amorphous white solid. 24: $\mathrm{R}_{f}=0.47$ (silica gel, EtOAc/hexanes, 1:1); IR (film) $v_{\max } 3318,2924,2106,1732,1532$, 1454, 1357, 1243, 1098, 1027, 738, $699 \mathrm{~cm}^{-1} ;{ }^{1} \mathrm{H}$ NMR (600 MHz, $\left.\mathrm{CDCl}_{3}\right) \delta$ 7.26-7.15 (m, 15 H), 5.42 (br s, 1 H), 5.27 (br m, 1 H), 4.65-4.47 (m, 5 H), 3.82 (m, 2 H), 3.74 (m, 2 H), 3.68 (s, 3 $\mathrm{H}) ;{ }^{13} \mathrm{C}$ NMR $\left(125 \mathrm{MHz}, \mathrm{CDCl}_{3}\right) \delta 155.7,138.0,137.6,137.1,128.6,128.4,128.3,128.1,128.0$, 127.9, 127.8, 127.6, 78.3, 73.8, 73.2, 72.8, 68.2, 60.0, 52.7, 53.4; HRMS (MALDI-FTMS) calcd for $\mathrm{C}_{34} \mathrm{H}_{38} \mathrm{NO}_{5}^{+}\left[\mathrm{M}+\mathrm{H}^{+}\right]$555.2214, found 555.2217.

Allylated sulfamidate $25 . \mathrm{Et}_{2} \mathrm{NH}(0.354 \mathrm{~mL}, 6.71 \mathrm{mmol}, 40$ equiv) was added to a solution of sulfamidate $23\left(0.100 \mathrm{~g}, 0.167 \mathrm{mmol}, 1.0\right.$ equiv) in $\mathrm{CH}_{3} \mathrm{CN} / \mathrm{H}_{2} \mathrm{O}(1: 1,3 \mathrm{~mL})$ at 25 ${ }^{\circ} \mathrm{C}$. After stirring this mixture for 5 minutes at $25{ }^{\circ} \mathrm{C}, \mathrm{Pd}(\mathrm{OAc})_{2}(0.002 \mathrm{~g}, 0.017 \mathrm{mmol}, 0.1$ equiv) and TPPTS (0.019 g, $0.033 \mathrm{mmol}, 0.2$ equiv) were added sequentially, providing a yellow solution which was stirred for an additional 30 minutes at $25{ }^{\circ} \mathrm{C}$. Upon completion, the reaction contents were poured into water $(5 \mathrm{~mL})$, and extracted with EtOAc $(3 \times 10 \mathrm{~mL})$. The combined organic layers were then dried $\left(\mathrm{MgSO}_{4}\right)$ and concentrated to give the desired deprotected sulfamidate $\left(0.075 \mathrm{~g}, 87 \%\right.$ yield) as a white oil. ${ }^{2}$ Pressing forward without any additional purification steps, this material $(0.075 \mathrm{~g}, 0.146 \mathrm{mmol}, 1.0$ equiv) was dissolved in DMSO (2 mL) and treated with $\mathrm{NaH}\left(0.029 \mathrm{~g}, 60 \%\right.$ dispersion in mineral oil, $0.730 \mathrm{mmol}, 5.0$ equiv) at $25^{\circ} \mathrm{C}$. After stirring the resultant reaction mixture for 10 minutes at $25{ }^{\circ} \mathrm{C}$, allyl bromide $(0.051 \mathrm{~mL}$, 
$0.584 \mathrm{mmol}, 4.0$ equiv) was added in a single portion; the reaction mixture was then stirred for an additional 15 minutes at $25^{\circ} \mathrm{C}$. Upon completion, the reaction contents were poured into water $(5 \mathrm{~mL})$, and extracted with EtOAc $(3 \times 10 \mathrm{~mL})$. The combined organic layers were then washed with water $(2 \times 15 \mathrm{~mL})$, dried $\left(\mathrm{MgSO}_{4}\right)$, and concentrated. The resultant light yellow oil was purified by flash column chromatography (silica gel, EtOAc/hexanes, $1: 3 \rightarrow 1: 1$ ) to give the allylated sulfamidate 25 (0.067 g, 73\% yield over two steps) as an amorphous white solid. 25: $\mathrm{R}_{f}$ $=0.64$ (silica gel, EtOAc/hexanes, 1:1); IR (film) $v_{\max } 2890,1452,1352,1193,1114,1052,988$, 741, 694, $606 \mathrm{~cm}^{-1} ;{ }^{1} \mathrm{H}$ NMR (600 MHz, $\left.\mathrm{CDCl}_{3}\right) \delta$ 7.30-7.24 (m, $\left.15 \mathrm{H}\right), 5.87$ (m, $\left.1 \mathrm{H}\right), 5.35$ (d, $J$ $=17.1 \mathrm{~Hz}, 1 \mathrm{H}), 5.29(\mathrm{~s}, 1 \mathrm{H}), 5.28(\mathrm{~d}, J=16.7 \mathrm{~Hz}, 1 \mathrm{H}), 4.95(\mathrm{dd}, J=7.4,5.3 \mathrm{~Hz}, 1 \mathrm{H}), 4.75$ $\left(\mathrm{AB}, J=11.0 \mathrm{~Hz}, \mathrm{v}_{\mathrm{ab}}=185.9 \mathrm{~Hz}, 2 \mathrm{H}\right), 4.73\left(\mathrm{AB}, J=11.8 \mathrm{~Hz}, v_{\mathrm{ab}}=76.7 \mathrm{~Hz}, 2 \mathrm{H}\right), 4.43(\mathrm{AB}, J$ $\left.=11.8 \mathrm{~Hz}, \mathrm{v}_{\mathrm{ab}}=26.3 \mathrm{~Hz}, 2 \mathrm{H}\right), 4.12(\mathrm{dd}, J=7.4,2.6 \mathrm{~Hz}, 1 \mathrm{H}), 4.04(\mathrm{t}, J=5.3 \mathrm{~Hz}, 1 \mathrm{H}), 4.00$ (app s, $1 \mathrm{H}), 3.79(\mathrm{dd}, J=14.5,5.7 \mathrm{~Hz}, 1 \mathrm{H}), 3.73(\mathrm{dd}, J=14.5,7.9 \mathrm{~Hz}, 1 \mathrm{H}), 3.52(\mathrm{AB}, J=9.6$ $\left.\mathrm{Hz}, \mathrm{v}_{\mathrm{ab}}=16.2 \mathrm{~Hz}, 2 \mathrm{H}\right) ;{ }^{13} \mathrm{C} \mathrm{NMR}\left(150 \mathrm{MHz}, \mathrm{CDCl}_{3}\right) \delta 137.8,137.6,137.4,130.4,128.5(2 \mathrm{C})$, 128.4, 128.2, 128.0 (2 C), 127.9, 127.7 (2 C), 121.1, 84.3, 82.2, 78.5, 74.9, 73.5 (2 C), 72.8, 72.4, 67.7, 47.0; HRMS (MALDI-FTMS) calcd for $\mathrm{C}_{30} \mathrm{H}_{33} \mathrm{NO}_{7} \mathrm{SNa}^{+}\left[\mathrm{M}+\mathrm{Na}^{+}\right]$574.1870, found 574.1866.

2,3,4-Tri- $\boldsymbol{O}$-benzyl-D-fucose (30). Concentrated $\mathrm{H}_{2} \mathrm{SO}_{4}(1 \mathrm{~mL})$ was added to a solution of D-fucose (1.64 g, $10.0 \mathrm{mmol}, 1.0$ equiv) in $\mathrm{MeOH}(30 \mathrm{~mL})$ at $25^{\circ} \mathrm{C}$, and the resultant mixture was stirred for 5 hours. Upon completion, the reaction contents were neutralized by the addition of $3 \mathrm{~N}$ aqueous $\mathrm{NaOH}$ (monitered using standard $\mathrm{pH}$ paper) and concentrated directly. The resulting white solid was then taken up in EtOAc $(50 \mathrm{~mL})$, filtered through a short silica gel, and concentrated to give 1-O-methyl-D-fucose (1.78 g, 100\% yield) as a white solid. Pressing forward without any additional purification steps, this newly-formed material (1.78 $\mathrm{g}, 10.0$ mmol, 1.0 equiv) was dissolved in DMF $(10 \mathrm{~mL})$ and treated with $\mathrm{NaH}(3.60 \mathrm{~g}, 60 \%$ dispersion in mineral oil, $90.0 \mathrm{mmol}, 9.0$ equiv) at $25^{\circ} \mathrm{C}$. After stirring the resultant solution for 5 minutes at $25{ }^{\circ} \mathrm{C}$, the reaction contents were cooled to $0{ }^{\circ} \mathrm{C}$ and benzyl bromide $(5.95 \mathrm{~mL}, 50.0 \mathrm{mmol}$, 5.0 equiv) added dropwise over the course of 10 minutes. The reaction mixture was then 
warmed to $25^{\circ} \mathrm{C}$ over the course of 4 hours. Upon completion, the reaction mixture was poured into $1 \mathrm{~N}$ aqueous $\mathrm{HCl}(25 \mathrm{~mL})$ and extracted with $\mathrm{Et}_{2} \mathrm{O}(3 \times 25 \mathrm{~mL})$. The combined organic layers were then washed with water $(2 \times 15 \mathrm{~mL})$, dried $\left(\mathrm{MgSO}_{4}\right)$, and concentrated. The resultant light yellow solid was purified by flash column chromatography (silica gel, $\mathrm{Et}_{2} \mathrm{O} /$ hexanes, $\left.1: 3 \rightarrow 1: 1\right)$ to give the desired tri- $O$-benzylated D-fucose intermediate $(3.7 \mathrm{~g}, 82 \%)$ as an amorphous white solid. Finally, a portion of this newly-formed product $(0.452 \mathrm{~g}, 1.0$ mmol, 1.0 equiv) was dissolved $6 \mathrm{M}$ aqueous $\mathrm{HCl} /$ concentrated $\mathrm{HCl}(1: 1,5 \mathrm{~mL})$ at $25{ }^{\circ} \mathrm{C}$. The resultant mixture was then warmed to $60{ }^{\circ} \mathrm{C}$ and stirred for 12 hours. Upon completion, the reaction contents were concentrated directly. The resultant yellow oil was purified by flash column chromatography (silica gel, $\mathrm{Et}_{2} \mathrm{O} /$ hexanes, $1: 1$ ) to give the desired D-fucose-derived starting material $30(0.297 \mathrm{~g}, 68 \%)$ as an amorphous white solid. 30: $\mathrm{R}_{f}=0.54$ (silica gel, EtOAc/hexanes, 1:1); IR (film) $v_{\max } 3416,3031,2867,1453,1364,1208,1098,740,698 \mathrm{~cm}^{-1}$; ${ }^{1} \mathrm{H}$ NMR (500 MHz, $\mathrm{CDCl}_{3}, 1: 1$ mixture of anomers) $\delta 7.48-7.34(\mathrm{~m}, 30 \mathrm{H}), 5.55(\mathrm{~s}, 1 \mathrm{H}), 5.41$ $(\mathrm{dd}, J=9.5,4.4 \mathrm{~Hz}, 1 \mathrm{H}), 4.83-4.49(\mathrm{~m}, 11 \mathrm{H}), 4.35($ app t, $J=5.2 \mathrm{~Hz}, 1 \mathrm{H}), 4.30$ (br d, $J=11.4$ $\mathrm{Hz}, 1 \mathrm{H}), 4.25(\operatorname{app~t}, J=5.2 \mathrm{~Hz}, 1 \mathrm{H}), 4.13(\mathrm{dd}, J=5.5,4.8 \mathrm{~Hz}, 1 \mathrm{H}), 4.10(\mathrm{dd}, J=2.2,1.1 \mathrm{~Hz}$, $1 \mathrm{H}), 4.05(\mathrm{dd}, J=5.2,2.2 \mathrm{~Hz}, 1 \mathrm{H}), 3.99(\mathrm{dd}, J=4.8,3.0 \mathrm{~Hz}, 1 \mathrm{H}), 3.78(\mathrm{~m}, 1 \mathrm{H}), 3.63(\mathrm{dd}, J=$ 6.3, 3.0 Hz, 1 H), 3.55 (br s, $1 \mathrm{H}), 3.48$ (m, $1 \mathrm{H}), 1.38$ (d, J=4.6 Hz, $3 \mathrm{H}), 1.28$ (d, $J=4.8 \mathrm{~Hz}, 3$ $\mathrm{H}) ;{ }^{13} \mathrm{C}$ NMR (125 MHz, $\mathrm{CDCl}_{3}, 1: 1$ mixture of anomers) $\delta 138.5,137.7,137.5,137.4(2 \mathrm{C})$, 137.3, 128.4 (2 C), 128.3 (2 C), 128.2, 128.0, 127.9 (2 C), 127.8 (3 C), 127.7, 127.5, 127.4, $126.9,100.7,96.0,87.3,85.2,84.5,84.4,82.8,82.2,74.0,73.9,71.9$ (2 C), 71.8, 71.6, 71.2, 71.1, 65.1; MS (ESI) calcd for $\mathrm{C}_{27} \mathrm{H}_{30} \mathrm{O}_{9} \mathrm{Cl}^{-}\left[\mathrm{M}+\mathrm{Cl}^{-}\right] 470$, found 470 .

2,3,4,6-Tetra- $\boldsymbol{O}$-methyl-D-mannose (32). D-Mannose (1.80 g, $10.0 \mathrm{mmol})$ was converted into $\mathbf{3 2}$ in a manner similar to the preparation of $\mathbf{3 0}$ by substituting methyl iodide for benzyl bromide, ultimately providing compound 32 (0.146 g, 62\% yield) as a white amorphous solid. 32: $\mathrm{R}_{f}=0.15$ (silica gel, EtOAc/hexanes, 1:2); IR (film) $v_{\max } 3402,2932,2828,1644$, 1453, 1193, 1109, 1030, 958, $792 \mathrm{~cm}^{-1} ;{ }^{1} \mathrm{H}$ NMR (500 MHz, $\left.\mathrm{CDCl}_{3}\right) \delta 5.25$ (s, $\left.1 \mathrm{H}\right), 4.62$ (br s, 1 H), $3.86(\mathrm{td}, J=7.3,1.8 \mathrm{~Hz}, 1 \mathrm{H}), 3.59-3.51(\mathrm{~m}, 4 \mathrm{H}), 3.45(\mathrm{~s}, 3 \mathrm{H}), 3.44(\mathrm{~s}, 3 \mathrm{H}), 3.43(\mathrm{~s}, 3 \mathrm{H})$, 
$3.32(\mathrm{~s}, 3 \mathrm{H}), 3.26(\mathrm{t}, J=9.2 \mathrm{~Hz}, 1 \mathrm{H}) ;{ }^{13} \mathrm{C}$ NMR $\left(125 \mathrm{MHz}, \mathrm{CDCl}_{3}\right) \delta$ 91.5, 80.8, 74.5, 77.1, 72.7, 70.3, 60.6, 59.1, 58.8, 57.6; HRMS (MALDI-FTMS) calcd for $\mathrm{C}_{10} \mathrm{H}_{20} \mathrm{O}_{6} \mathrm{Na}^{+}\left[\mathrm{M}+\mathrm{Na}^{+}\right]$ 259.1152 , found 259.1152 .

2,3,5-Tri-O-benzyl-L-arabinofuranose 34. Lactol 34 was prepared from L-arabinose using the sequence established by Tejima and Fletcher. ${ }^{3}$ 34: $\mathrm{R}_{f}=0.51$ (silica gel, EtOAc/hexanes, 1:1); IR (film) $v_{\max } 3418,3030,2866,1495,1454,1365,1264,1208,1099$, 1028, 738, $698 \mathrm{~cm}^{-1} ;{ }^{1} \mathrm{H}$ NMR (500 MHz, $\mathrm{CDCl}_{3}, 1: 1$ mixture of anomers) $\delta$ 7.36-7.26 (m, 30 H), $5.40(\mathrm{~s}, 1 \mathrm{H}), 5.34(\mathrm{~d}, J=3.7 \mathrm{~Hz}, 1 \mathrm{H}), 4.66(\mathrm{~d}, J=9.5 \mathrm{~Hz}, 1 \mathrm{H}), 4.61-4.52(\mathrm{~m}, 10 \mathrm{H}), 4.48-$ $4.45(\mathrm{~m}, 2 \mathrm{H}), 4.17(\mathrm{t}, J=4.0 \mathrm{~Hz}, 1 \mathrm{H}), 4.09$ (q, $J=3.7 \mathrm{~Hz}, 1 \mathrm{H}), 4.02(\mathrm{t}, J=4.0 \mathrm{~Hz}, 1 \mathrm{H}), 3.98$ $(\mathrm{d}, J=1.5 \mathrm{~Hz}, 1 \mathrm{H}), 3.94(\mathrm{~m}, 1 \mathrm{H}), 3.61-3.50(\mathrm{~m}, 4 \mathrm{H}),{ }^{13} \mathrm{C} \mathrm{NMR}\left(125 \mathrm{MHz}, \mathrm{CDCl}_{3}, 1: 1\right.$ mixture of anomers) $\delta 138.0,137.4,137.3,137.2$ (2 C), 128.6, 128.5 (4 C), 128.4 (2 C), 128.3, 128.0 (2 C), 127.9 (2 C), 127.8 (2 C), 127.7 (2 C), 127.6, 101.1, 96.2, 86.3, 84.0, 82.6, 82.0, 81.8, 80.5, 76.8, 73.5, 73.3, 72.2, 72.0 (2 C), 71.7, 70.4, 70.1; HRMS (MALDI-FTMS) calcd for $\mathrm{C}_{26} \mathrm{H}_{28} \mathrm{O}_{5} \mathrm{Na}^{+}\left[\mathrm{M}+\mathrm{Na}^{+}\right]$443.1829, found 443.1809.

Furanose 36. The requisite lactol intermediate was prepared from D-ribose following the procedure of Kaskar and co-workers. ${ }^{4} \quad$ 36: $\mathrm{R}_{f}=0.51$ (silica gel, EtOAc/hexanes, 1:2); IR (film) $v_{\max } 3416,2934,2858,1469,1381,1257,1211,1075,1004,939,838,779 \mathrm{~cm}^{-1} ;{ }^{1} \mathrm{H}$ NMR $(500$ $\left.\mathrm{MHz}, \mathrm{CDCl}_{3}\right) \delta 5.24(\mathrm{~d}, J=11.4 \mathrm{~Hz}, 1 \mathrm{H}), 4.74(\mathrm{~d}, J=11.7 \mathrm{~Hz}, 1 \mathrm{H}), 4.66(\mathrm{~d}, J=5.9 \mathrm{~Hz}, 1 \mathrm{H})$, 4.47 (d, $J=5.9 \mathrm{~Hz}, 1 \mathrm{H}), 4.31$ (s, $1 \mathrm{H}), 3.75-3.72$ (m, 2 H), 1.45 (s, $3 \mathrm{H}), 1.29$ (s, $3 \mathrm{H}), 0.90$ (s, 9 H), $0.11(\mathrm{~s}, 3 \mathrm{H}), 0.10$ (s, $3 \mathrm{H}),{ }^{13} \mathrm{C}$ NMR $\left(100 \mathrm{MHz}, \mathrm{CDCl}_{3}\right) \delta$ 111.9, 103.3, 87.5, 86.8, 81.7, 64.7, 26.4, 25.7, 24.8, 18.1 -5.7; HRMS (MALDI-FTMS) calcd for $\mathrm{C}_{14} \mathrm{H}_{28} \mathrm{O}_{5} \mathrm{SiNa}^{+}\left[\mathrm{M}+\mathrm{Na}^{+}\right]$ 327.1598 , found 327.1592 .

General procedure for the synthesis of $\beta$-disposed glycosylamines. The lactol starting material (0.5 mmol, 1.0 equiv) was dissolved in $\mathrm{THF} / \mathrm{CH}_{2} \mathrm{Cl}_{2}(4: 1,5 \mathrm{~mL})$ and the desired Burgess-type reagent $\left(\mathbf{1}, \mathbf{2}\right.$, or $\mathbf{3}, 0.75 \mathrm{mmol}, 1.5$ equiv) was added at $25^{\circ} \mathrm{C}$ in a single portion. The resultant solution was immediately warmed to reflux (using a preheated oil bath) and stirred for 6 hours. Upon completion, the reaction contents were cooled to $25^{\circ} \mathrm{C}$, poured into saturated 
aqueous $\mathrm{NH}_{4} \mathrm{Cl}(25 \mathrm{~mL})$, and extracted with $\mathrm{CH}_{2} \mathrm{Cl}_{2}(3 \times 25 \mathrm{~mL})$. The combined organic layers were then washed with water $(50 \mathrm{~mL})$, dried $\left(\mathrm{MgSO}_{4}\right)$, and concentrated. The resultant yellow residue was purified by flash column chromatography (silica gel) in an appropriate solvent system to give the desired product in high purity.

$\mathrm{CO}_{2} \mathrm{Me}$-protected 2,3,4,6-tetra- $O$-methyl- $\beta$-D-glucosylamine (27): $\mathrm{R}_{f}=0.13$ (silica gel, EtOAc/hexanes, 1:1); IR (film) $v_{\max } 3329,2937,2836,1712,1544,1450,1278,1158,1099$, 989, 945, $647 \mathrm{~cm}^{-1} ;{ }^{1} \mathrm{H}$ NMR $\left(400 \mathrm{MHz}, \mathrm{CDCl}_{3}\right) \delta 5.30(\mathrm{~d}, J=8.8 \mathrm{~Hz}, 1 \mathrm{H}), 4.75(\mathrm{t}, J=8.4 \mathrm{~Hz}$, $1 \mathrm{H}), 3.68$ (br s, $3 \mathrm{H}), 3.62(\mathrm{~s}, 3 \mathrm{H}), 3.58-3.53(\mathrm{~m}, 2 \mathrm{H}), 3.52(\mathrm{~s}, 3 \mathrm{H}), 3.51(\mathrm{~s}, 3 \mathrm{H}), 3.41(\mathrm{~s}, 3 \mathrm{H})$, $3.30(\mathrm{~m}, 1 \mathrm{H}), 3.22(\mathrm{~d}, J=6.7 \mathrm{~Hz}, 2 \mathrm{H}), 2.91$ (br m, $1 \mathrm{H}) ;{ }^{13} \mathrm{C} \mathrm{NMR}\left(100 \mathrm{MHz}, \mathrm{CDCl}_{3}\right) \delta 156.1$, 99.5, 87.2, 82.8, 81.6, 78.9, 75.8, 70.5, 60.8, 60.3, 59.1, 52.4; HRMS (MALDI-FTMS) calcd for $\mathrm{C}_{12} \mathrm{H}_{23} \mathrm{NO}_{7} \mathrm{Na}^{+}\left[\mathrm{M}+\mathrm{Na}^{+}\right] 316.1367$, found 316.1363.

Figure S1. X-ray crystal structure of compound 27.

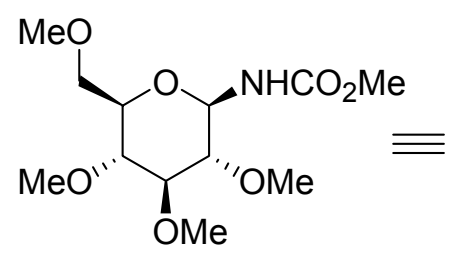

27

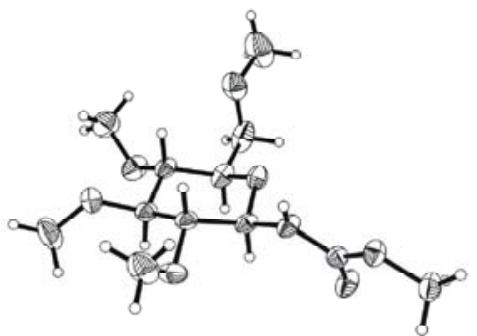

Alloc-protected 2,3,4,6-tetra- $O$-benzyl- $\beta$-D-glucosylamine (29): $\mathrm{R}_{f}=0.64$ (silica gel, EtOAc/hexanes, 1:1); IR (film) $v_{\max } 3331,3030,2867,1703,1537,1453,1360,1280,1071,735$, $697 \mathrm{~cm}^{-1} ;{ }^{1} \mathrm{H}$ NMR $\left(600 \mathrm{MHz}, \mathrm{CDCl}_{3}\right) \delta$ 7.45-7.32 (m, $\left.18 \mathrm{H}\right), 7.19-7.17$ (m, $\left.2 \mathrm{H}\right), 5.93$ (ddd, $J$ $=22.7,10.9,5.7 \mathrm{~Hz}, 1 \mathrm{H}), 5.32(\mathrm{~d}, J=17.1 \mathrm{~Hz}, 1 \mathrm{H}), 5.23(\mathrm{~d}, J=10.1 \mathrm{~Hz}, 1 \mathrm{H}), 5.07(\mathrm{~d}, J=10.1$ $\mathrm{Hz}, 1 \mathrm{H}), 4.92(\mathrm{~m}, 1 \mathrm{H}), 4.91\left(\mathrm{AB}, J=11.0 \mathrm{~Hz}, \mathrm{v}_{\mathrm{ab}}=18.8 \mathrm{~Hz}, 2 \mathrm{H}\right), 4.82(\mathrm{~d}, J=10.9 \mathrm{~Hz}, 2 \mathrm{H})$, $4.72(\mathrm{~d}, J=11.0 \mathrm{~Hz}, 1 \mathrm{H}), 4.64-4.60(\mathrm{~m}, 3 \mathrm{H}), 4.53(\mathrm{~d}, J=10.5 \mathrm{~Hz}, 1 \mathrm{H}), 4.47(\mathrm{~d}, J=12.3 \mathrm{~Hz}, 1$ $\mathrm{H}), 3.73(\mathrm{~m}, 4 \mathrm{H}), 3.52(\mathrm{~d}, J=7.9 \mathrm{~Hz}, 1 \mathrm{H}), 3.38(\mathrm{t}, J=8.3 \mathrm{~Hz}, 1 \mathrm{H}) ;{ }^{13} \mathrm{C} \mathrm{NMR}(150 \mathrm{MHz}$, $\left.\mathrm{CDCl}_{3}\right) \delta 155.3,138.3,138.0,137.7,132.4,128.5,128.4(3 \mathrm{C}), 128.3(3 \mathrm{C}), 128.2,128.0,127.8$ 
127.7, 127.6, 117.8, 85.9, 81.6, 80.1, 77.4, 76.1, 75.6, 74.9, 74.7, 73.5, 68.1; HRMS (MALDIFTMS) calcd for $\mathrm{C}_{38} \mathrm{H}_{41} \mathrm{NO}_{7} \mathrm{Na}^{+}\left[\mathrm{M}+\mathrm{Na}^{+}\right] 646.2775$, found 646.2766 .

$\mathrm{CO}_{2}$ Me-protected 2,3,4-tri- $O$-benzyl- $\beta$-D-fucosylamine (31): $\mathrm{R}_{f}=0.70$ (silica gel, EtOAc/hexanes, 1:1); IR (film) $v_{\max } 3326,2927,1730,1504,1453,1351,1067,736,697 \mathrm{~cm}^{-1}$; ${ }^{1} \mathrm{H}$ NMR (400 MHz, $\left.\mathrm{CDCl}_{3}\right) \delta 7.36-7.31(\mathrm{~m}, 15 \mathrm{H}), 5.81$ (br d, $\left.J=6.4 \mathrm{~Hz}, 1 \mathrm{H}\right), 5.76$ (br s, $1 \mathrm{H}$ ), $4.69\left(\mathrm{AB}, J=9.4 \mathrm{~Hz}, v_{\mathrm{ab}}=21.4 \mathrm{~Hz}, 2 \mathrm{H}\right), 4.67(\mathrm{~m}, 1 \mathrm{H}), 4.57(\mathrm{~m}, 3 \mathrm{H}), 4.24(\mathrm{dd}, J=4.7,2.6 \mathrm{~Hz}$, $1 \mathrm{H}), 4.04(\mathrm{~d}, J=1.5 \mathrm{~Hz}, 1 \mathrm{H}), 3.98(\mathrm{br} \mathrm{s}, 1 \mathrm{H}), 3.80(\mathrm{~m}, 1 \mathrm{H}), 3.77(\mathrm{br} \mathrm{s}, 3 \mathrm{H}), 1.20(\mathrm{~d}, J=4.9$ $\mathrm{Hz}, 3 \mathrm{H}) ;{ }^{13} \mathrm{C} \mathrm{NMR}\left(100 \mathrm{MHz}, \mathrm{CDCl}_{3}\right) \delta 155.8,138.6,137.2,128.5,128.4,128.3,128.0,127.9$, 127.8 (2 C), 127.6, 127.4, 86.1, 85.3 (2 C), 82.4, 74.1, 71.8, 71.4, 52.3, 15.7; HRMS (MALDIFTMS) calcd for $\mathrm{C}_{29} \mathrm{H}_{33} \mathrm{NO}_{6} \mathrm{Na}^{+}\left[\mathrm{M}+\mathrm{Na}^{+}\right] 514.2200$, found 514.2191.

Alloc-protected 2,3,4,6-tetra- $\boldsymbol{O}$-methyl- $\beta$-D-mannosylamine (33): $\mathrm{R}_{f}=0.38$ (silica gel, EtOAc); IR (film) $v_{\max } 3332,2934,1726,1529,1450,1305,1237,1100 \mathrm{~cm}^{-1} ;{ }^{1} \mathrm{H}$ NMR $(500$ $\left.\mathrm{MHz}, \mathrm{CDCl}_{3}\right) \delta 5.90(\mathrm{ddd}, J=23.1,10.7,4.8 \mathrm{~Hz}, 1 \mathrm{H}), 5.52(\mathrm{br} \mathrm{s}, 1 \mathrm{H}), 5.32(\mathrm{~d}, J=17.2 \mathrm{~Hz}, 1$ H), $5.22(\mathrm{~d}, J=10.3 \mathrm{~Hz}, 1 \mathrm{H}), 4.60$ (s, $2 \mathrm{H}), 3.63-3.48(\mathrm{~m}, 15 \mathrm{H}), 3.45-3.42(\mathrm{~m}, 4 \mathrm{H}) ;{ }^{13} \mathrm{C} \mathrm{NMR}$ $\left(100 \mathrm{MHz}, \mathrm{CDCl}_{3}\right) \delta 155.5,132.7,118.7,80.4,77.1,76.4,73.3,71.6,66.5,59.7,58.8,58.3$, 51.5; HRMS (MALDI-FTMS) calcd for $\mathrm{C}_{14} \mathrm{H}_{25} \mathrm{NO}_{7} \mathrm{Na}^{+}\left[\mathrm{M}+\mathrm{Na}^{+}\right] 342.1523$, found 342.1522 .

$\mathrm{CO}_{2}$ Me-protected 2,3,5-tri- $O$-benzyl- $\beta$-L-arabinofuranosylamine 35: $\mathrm{R}_{f}=0.63$ (silica gel, EtOAc/hexanes, 1:1); IR (film) $v_{\max } 3317,3030,2865,1732,1498,1454,1363,1238,1058$, 739, $698 \mathrm{~cm}^{-1} ;{ }^{1} \mathrm{H}$ NMR (400 MHz, $\left.\mathrm{CDCl}_{3}\right) \delta 7.35-7.27(\mathrm{~m}, 15 \mathrm{H}), 5.88($ br d, $J=9.4 \mathrm{~Hz}, 1 \mathrm{H}$ ), $5.76($ br d, $J=10.0 \mathrm{~Hz}, 1 \mathrm{H}), 4.61-4.45(\mathrm{~m}, 6 \mathrm{H}), 4.38($ app t, $J=5.6 \mathrm{~Hz}, 1 \mathrm{H}), 4.02(\mathrm{~s}, 1 \mathrm{H})$, $3.93(\mathrm{~s}, 1 \mathrm{H}), 3.70(\mathrm{br} \mathrm{s}, 3 \mathrm{H}), 3.64(\mathrm{dd}, J=9.4,5.9 \mathrm{~Hz}, 1 \mathrm{H}), 3.52(\operatorname{app~t}, J=8.8 \mathrm{~Hz}, 1 \mathrm{H}) ;{ }^{13} \mathrm{C}$ NMR (100 MHz, $\left.\mathrm{CDCl}_{3}\right) \delta 155.8,137.9,137.1,128.4$ (2 C), 128.3, 128.2, 127.9, 127.8, 127.7, $127.692 \mathrm{C}$ ), 85.5, 84.9, 82.7, 82.1, 73.2, 71.6, 71.4, 70.0, 52.2; HRMS (MALDI-FTMS) calcd for $\mathrm{C}_{28} \mathrm{H}_{31} \mathrm{NO}_{6} \mathrm{Na}^{+}\left[\mathrm{M}+\mathrm{Na}^{+}\right]$500.2043, found 500.2033.

$\mathrm{CO}_{2}$ Me-protected ribosylamine 37: $\mathrm{R}_{f}=0.67$ (silica gel, EtOAc/hexanes, 1:2); IR (film) $v_{\max } 3347,2933,1737,1509,1461,1377,1251,1082,931,837,779 \mathrm{~cm}^{-1} ;{ }^{1} \mathrm{H}$ NMR (400 $\left.\mathrm{MHz}, \mathrm{CDCl}_{3}\right) \delta 6.27$ (br d, $\left.J=11.8 \mathrm{~Hz}, 1 \mathrm{H}\right), 5.68($ br d, $J=10.0 \mathrm{~Hz}, 1 \mathrm{H}), 4.72(\mathrm{~d}, J=5.9 \mathrm{~Hz}, 1$ 
H), $4.50(\mathrm{~d}, J=6.2 \mathrm{~Hz}, 1 \mathrm{H}), 4.29(\mathrm{~s}, 1 \mathrm{H}), 3.77(\mathrm{~m}, 1 \mathrm{H}), 3.71(\mathrm{~m}, 3 \mathrm{H}), 3.66$ (br s, $3 \mathrm{H}), 1.51$ (s, $3 \mathrm{H}), 1.33(\mathrm{~s}, 3 \mathrm{H}), 0.94(\mathrm{~s}, 9 \mathrm{H}), 0.13(\mathrm{~s}, 6 \mathrm{H}) ;{ }^{13} \mathrm{C} \mathrm{NMR}\left(100 \mathrm{MHz}, \mathrm{CDCl}_{3}\right) \delta 155.8,112.2$, 89.0, 86.6, 86.0, 82.1, 79.4, 65.0, 51.8, 25.7, 24.8, 18.1; HRMS (MALDI-FTMS) calcd for $\mathrm{C}_{16} \mathrm{H}_{31} \mathrm{NO}_{6} \mathrm{SiNa}^{+}\left[\mathrm{M}+\mathrm{Na}^{+}\right]$384.1813, found 384.1814.

2,3,4,6-Tetra-O-benzyl- $\boldsymbol{\beta}$-D-glucosylamine. $\mathrm{Et}_{2} \mathrm{NH}(0.179 \mathrm{~mL}, 3.40 \mathrm{mmol}, 40$ equiv) was added to a solution of intermediate 29 (0.053 g, $0.085 \mathrm{mmol}, 1.0$ equiv) in $\mathrm{CH}_{3} \mathrm{CN} / \mathrm{H}_{2} \mathrm{O}(1: 1$, $2 \mathrm{~mL})$ at $25{ }^{\circ} \mathrm{C}$. After stirring this mixture for 5 minutes at $25^{\circ} \mathrm{C}, \mathrm{Pd}(\mathrm{OAc})_{2}(0.002 \mathrm{~g}, 0.009$ mmol, 0.1 equiv) and TPPTS (0.010 g, $0.017 \mathrm{mmol}, 0.2$ equiv) were added sequentially, providing a yellow solution which was stirred for an additional 30 minutes at $25{ }^{\circ} \mathrm{C}$. Upon completion, the reaction contents were poured into water $(5 \mathrm{~mL})$, and extracted with EtOAc $(3 \times$ $10 \mathrm{~mL})$. The combined organic layers were then dried $\left(\mathrm{MgSO}_{4}\right)$ and concentrated to give 2,3,4,6-tetra- $O$-benzyl- $\beta$-D-glucosylamine $(0.043 \mathrm{~g}, 95 \% \text { yield })^{2}$ as an amorphous white solid: $\mathrm{R}_{f}$ $=0.48$ (silica gel, EtOAc/hexanes, 1:1); IR (film) $v_{\max } 3352,3030,2862,1453,1351,1122$, 1094, 1086, 1027, 750, $698 \mathrm{~cm}^{-1} ;{ }^{1} \mathrm{H}$ NMR (600 MHz, $\left.\mathrm{CDCl}_{3}\right) \delta 7.48-7.21(\mathrm{~m}, 20 \mathrm{H}), 5.08(\mathrm{~d}, J$ $=13.2 \mathrm{~Hz}, 1 \mathrm{H}), 5.03(\mathrm{~d}, J=13.1 \mathrm{~Hz}, 1 \mathrm{H}), 4.94-4.86(\mathrm{~m}, 3 \mathrm{H}), 4.65\left(\mathrm{AB}, J=14.3 \mathrm{~Hz}, v_{\mathrm{ab}}=\right.$ $37.4 \mathrm{~Hz}, 2 \mathrm{H}), 4.58(\mathrm{~m}, 1 \mathrm{H}), 4.20(\mathrm{~d}, J=9.9 \mathrm{~Hz}, 1 \mathrm{H}), 3.85-3.80(\mathrm{~m}, 5 \mathrm{H}), 3.56(\mathrm{dd}, J=11.5$, $3.3 \mathrm{~Hz}, 1 \mathrm{H}), 3.30(\mathrm{t}, J=10.4 \mathrm{~Hz}, 1 \mathrm{H}), 1.97($ br s, $2 \mathrm{H}) ;{ }^{13} \mathrm{C} \mathrm{NMR}\left(150 \mathrm{MHz}, \mathrm{CDCl}_{3}\right) \delta 138.6$, 138.3, 138.0, 137.9, 128.7, 128.4 (2 C), 128.3, 128.2, 127.9, 127.8 (2 C), 127.7 (2 C), 127.6, 86.2, 85.8, 83.4, 78.1, 75.7, 75.6, 75.0, 74.9, 73.5, 68.9; HRMS (MALDI-FTMS) calcd for $\mathrm{C}_{34} \mathrm{H}_{38} \mathrm{NO}_{5}^{+}\left[\mathrm{M}+\mathrm{H}^{+}\right] 540.2744$, found $540.2750 .{ }^{5}$

\section{References}

1. Larsen, E; Jørgensen, P. T.; Sofan, M. A.; Pedersen, E. B. Synthesis 1994, 1037.

2. This procedure was modified from that established by Genêt, J. P.; Blart, E.; Savignac, M.; Lemeure, S.; Paris, J.-M. Tetrahedron Lett. 1993, 34, 4189.

3. Tejima, S.; Fletcher, H. G. J. Org. Chem. 1963, 28, 2999.

4. Kaskar, B.; Heise, G. L.; Michalak, R. S.; Vishnuvajjala, B. R. Synthesis 1990, 1031. 
5. The data for this amine is in full agreement with that published by Dorsey, A. D.; Barbarow, J. E.; Trauner, D. Org. Lett. 2003, 5, 3237. 

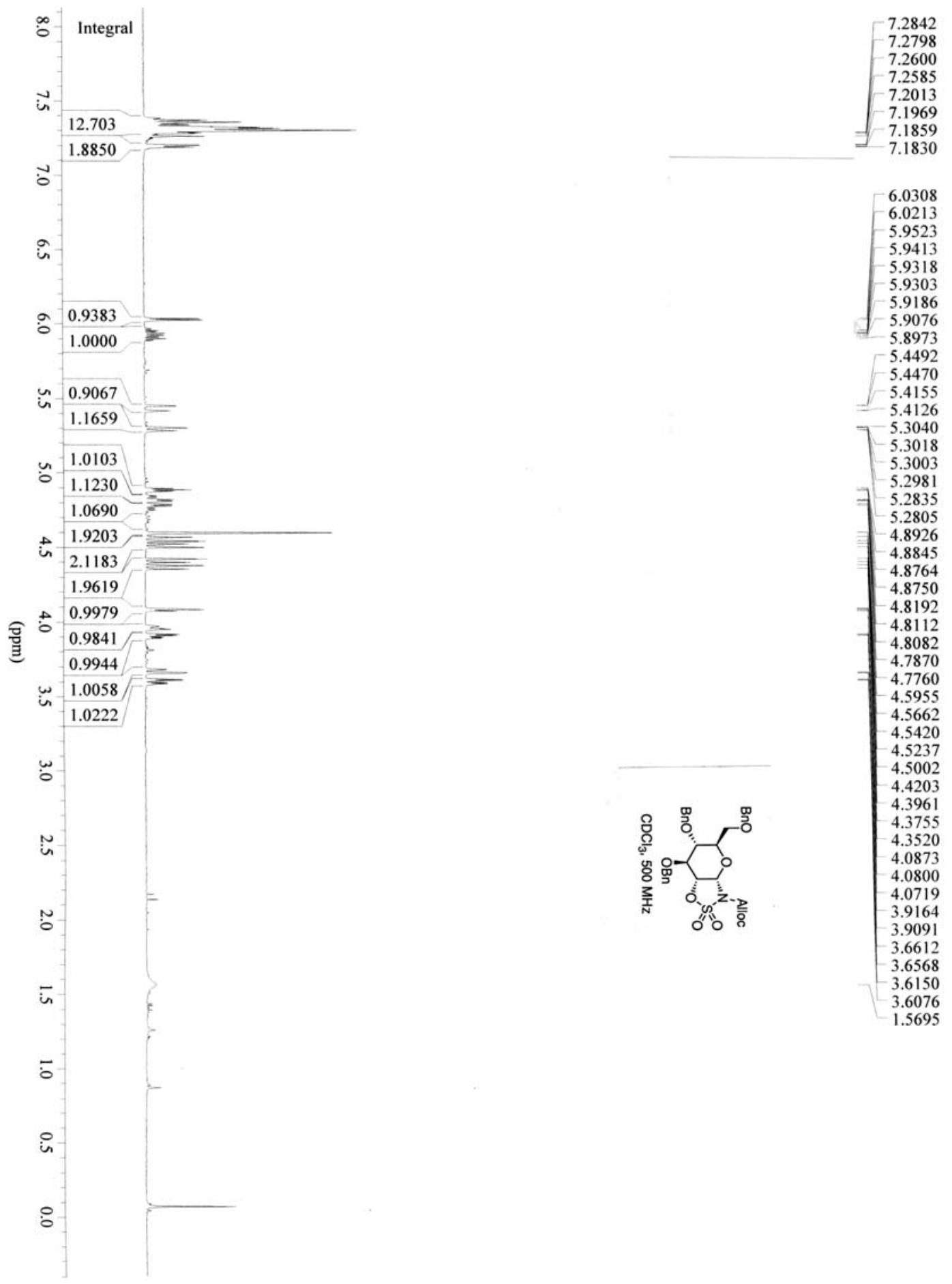


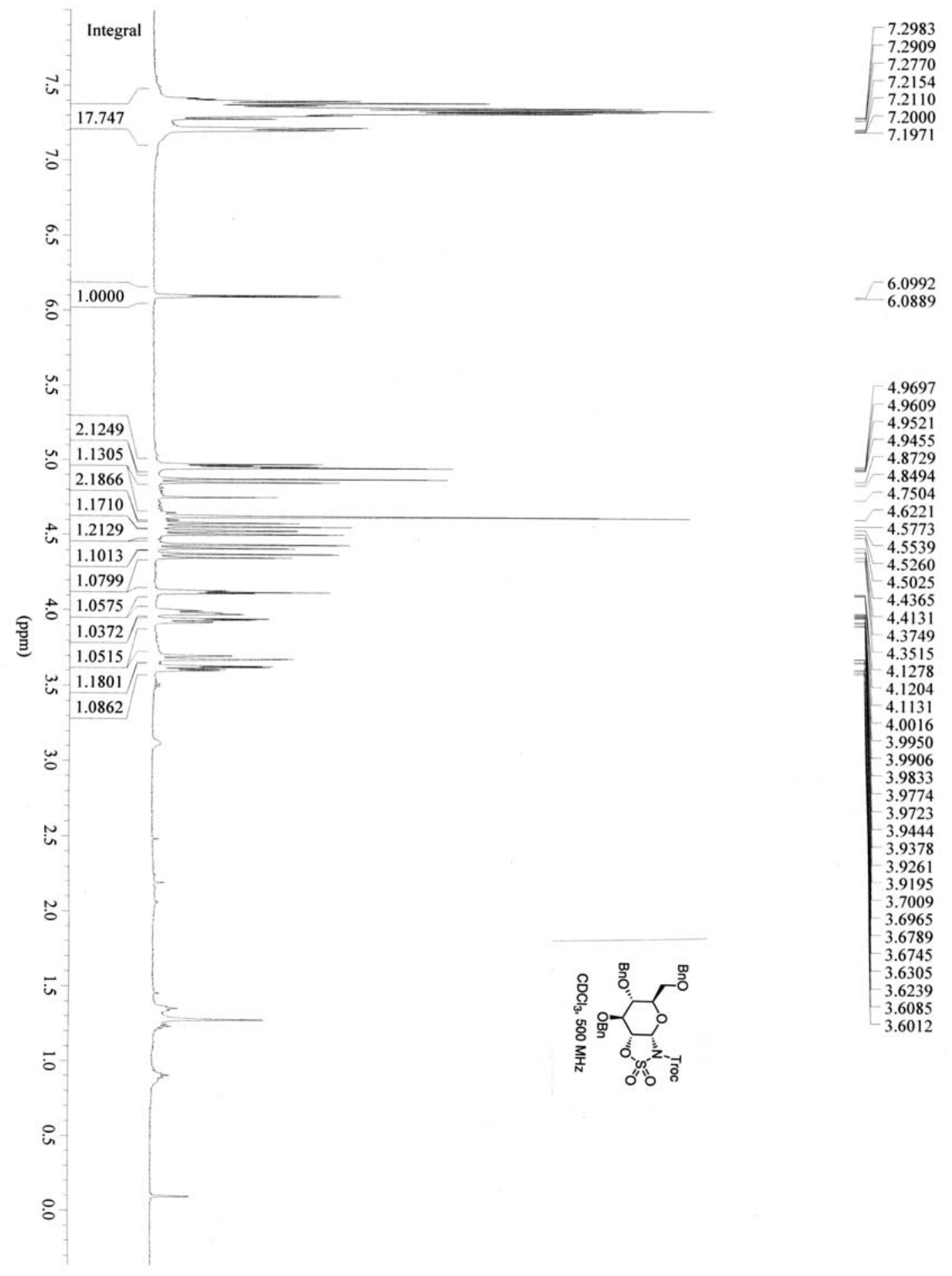




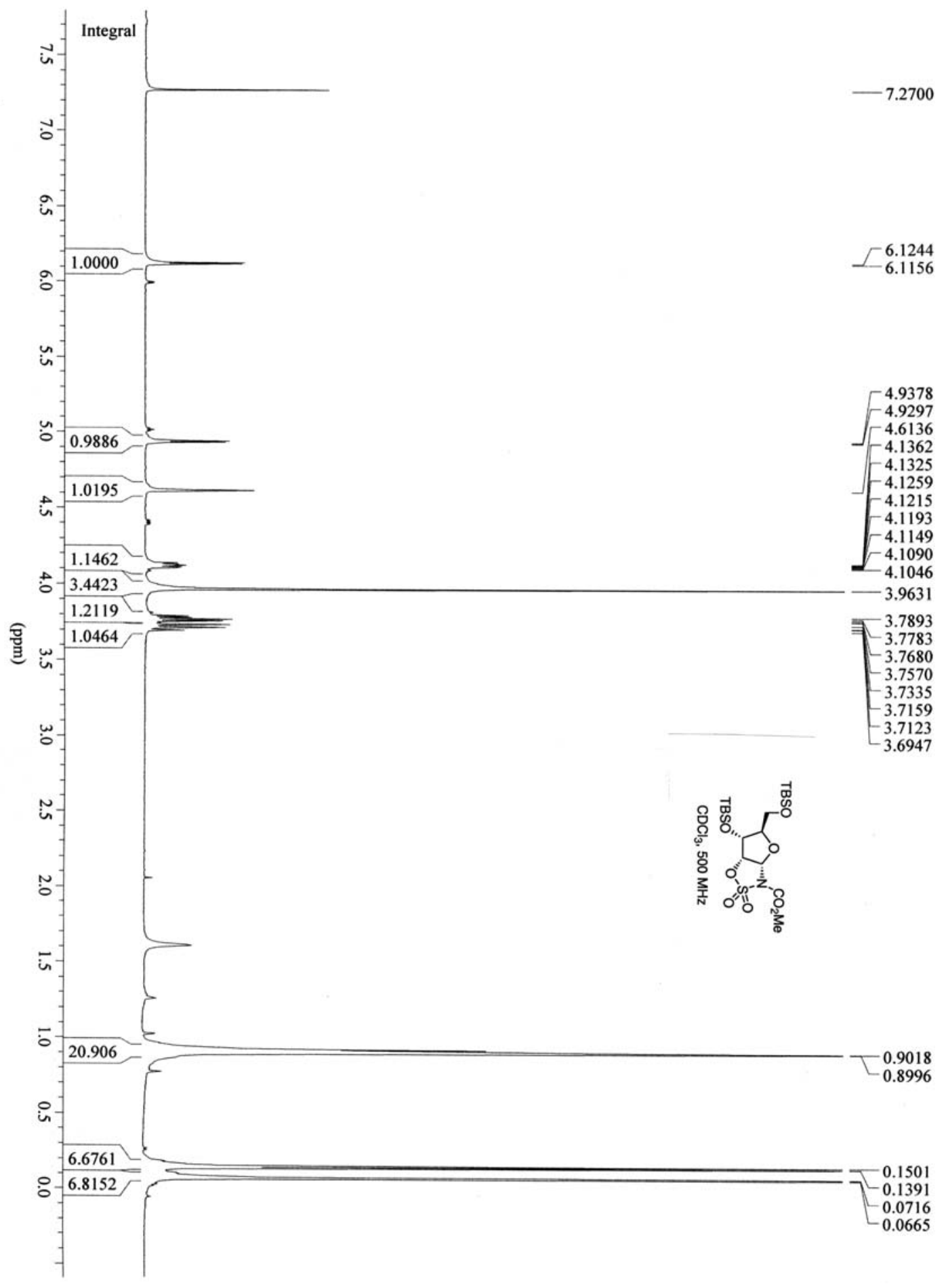




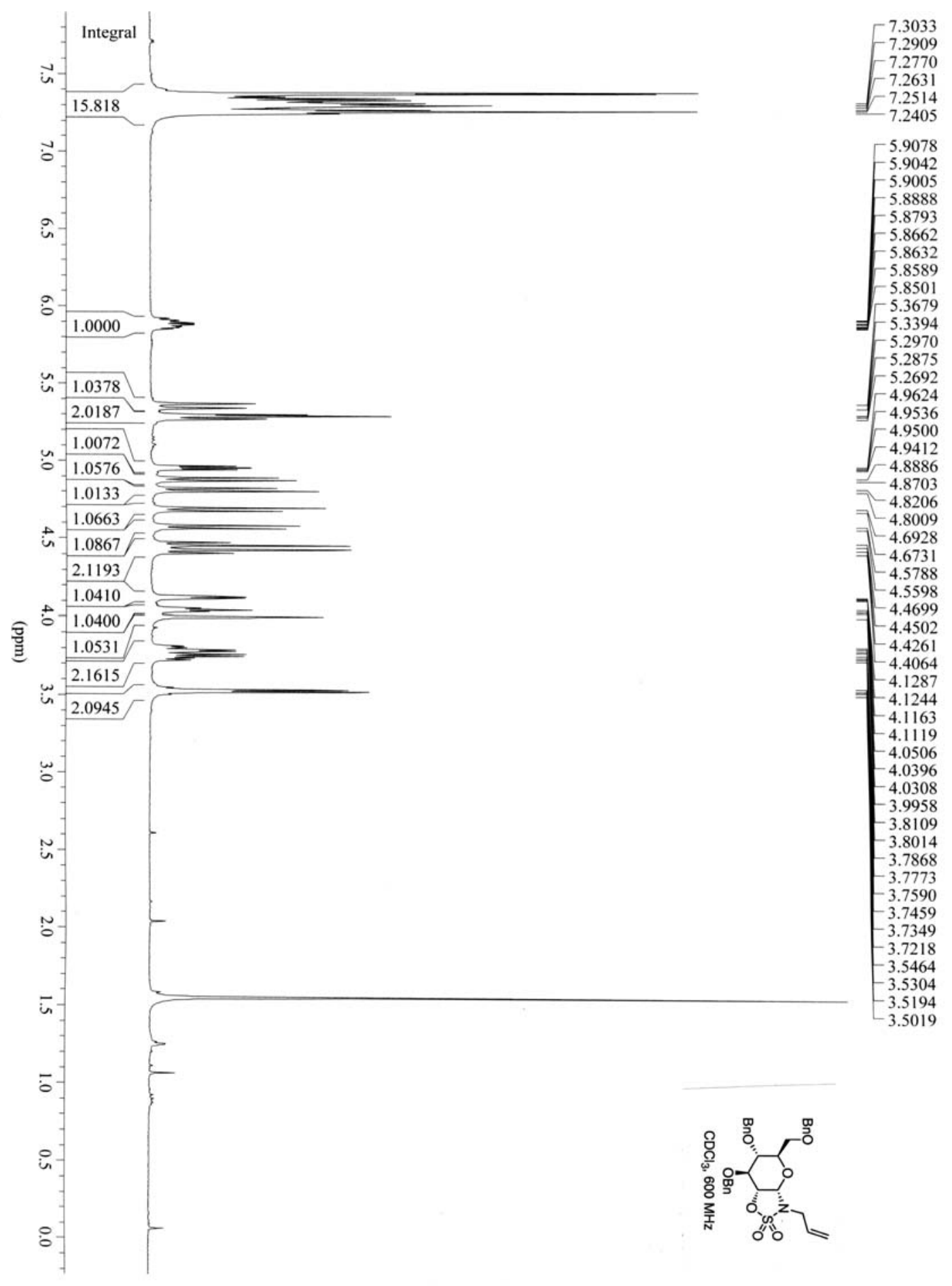




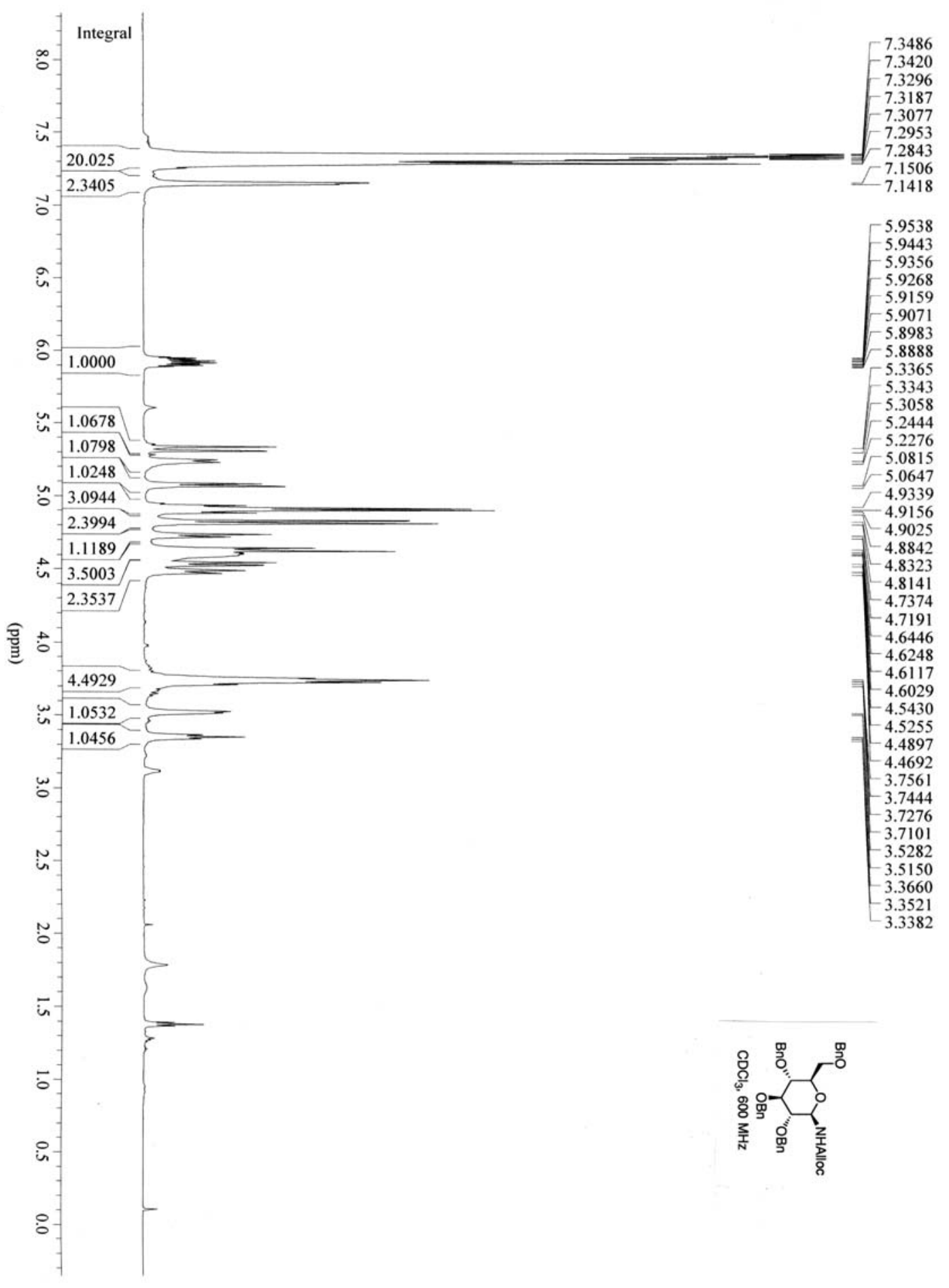




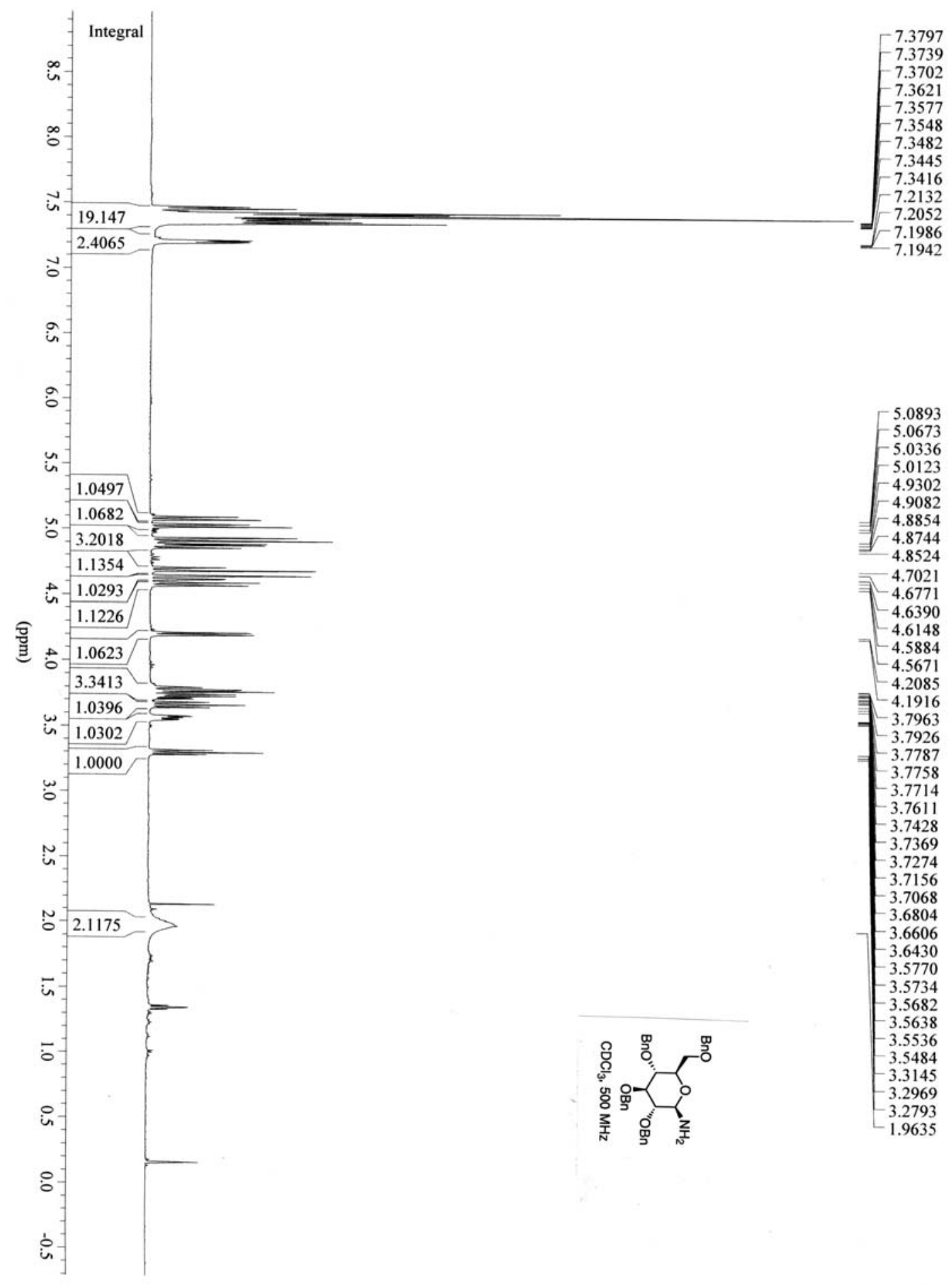

